\title{
Caracterización cíclica multidimensional de suelos no cohesivos
}

\author{
Characterization of multidimensional cycles of non cohesive soils
}

Fecha de entrega: 22 de noviembre 2014

Fecha de aceptación: 25 de mayo 2015

\section{Mauro Poblete ${ }^{1,2}$, Torsten Wichtmann ${ }^{2}$, Andrzej Niemunis ${ }^{2}$ y Theodoros Triantafyllidis ${ }^{2}$}

${ }^{1}$ Departamento de Ingeniería Civil, Universidad Católica de la Santísima Concepción, Alonso de Ribera 2850, Concepción, Chile, mauro@ucsc.cl

${ }^{2}$ Institute of Soil Mechanics and Rock Mechanics, Karlsruhe Institute of Technology KIT, Engler-Bunte Ring 14, 76131, Karlsruhe, Germany, mauro.freire@student.kit.edu, Torsten.Wichtmann@kit.edu,Andrzej.Niemunis@kit.edu,

Theodoros.Triantafyllidis@kit.edu

Las cargas con ciclos de deformación multidimensional pueden ser provocadas tanto por el trafico, como por cargas de viento y oleaje (en el caso de turbinas eólicas mar afuera por ejemplo), o porterremotos. El presente artículo se enfoca en la acumulación de deformaciones permanentes producto de carga cíclica de muchos ciclos, esto se refiere a una gran cantidad de ciclos de amplitud de deformación pequeña a mediana. Se provee evidencia experimental de ciclos de deformación complejos. Se presentan resultados de ensayos triaxiales cíclicos drenados con oscilación simultánea de la tensión axial y lateral. Se ensayan ciclos de deformación mediante la superposición de funciones armónicas con diferentes frecuencias y amplitudes. Para comparar, en un segundo ensayo se aplican las mismas oscilaciones pero separadas una a continuación de la otra. También se varía la secuencia de aplicación de las oscilaciones.

Palabras clave: carga cíclica, ciclos de deformación multidimensional, acumulación de deformación
A cyclic loading with multidimensional strain loops in the soil may be caused by traffic loading, wind and wave loading (e.g. offshore wind turbines) or by earthquake shaking. The present paper focuses on the accumulation of permanent deformations due to high-cyclic loading. That means loading with many cycles of small to intermediate strain amplitude. Experimental evidence for complicated strain loops is provided. Drained axisymmetric triaxial tests with a simultaneous oscillation of the axial and the lateral stress are presented. Strain loops obtained by a superposition of several harmonic functions with different frequencies and amplitudes are tested. For comparison, in a second test, the same oscillations are applied separately in succession. The sequence of application of the oscillations will also be varied.

Keywords: cyclic loading, multidimensional strain loops, strain accumulation

\section{Introducción}

Las cargas cíclicas pueden producir deformaciones permanentes en los suelos. Existen casos en que las cargas cíclicas, a las que puede ser sometida la fundación de una estructura, son del tipo multidimensional. Es el caso de las turbinas eólicas en que la carga de viento y la carga de las olas no necesariamente actúan en una misma dirección y por ende se transmiten a la fundación como una carga multidimensional. Sucede algo similar en las edificaciones cercanas a líneas férreas en que las vibraciones producidas por el paso de los trenes induce cargas cíclicas multidimensionales que pueden llegar a acumular grandes deformaciones en estructuras como estaciones de ferrocarril (Huber, 1988). Otro ejemplo de carga cíclica multidimensional son los sismos (Ishihara y Yamazaki, 1980). La Figura 1 muestra mediciones de aceleración multidimensional durante el terremoto de Niigata de 1964 y mediciones de ciclos de velocidad producidos por el tráfico. 
a)

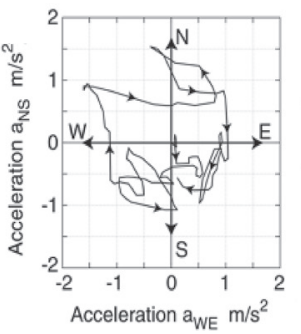

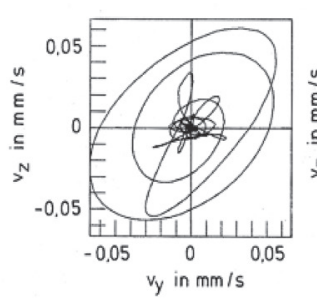

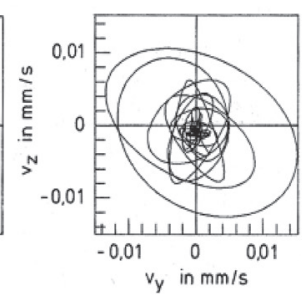

b)
Figura 1: a) Aceleración multidimensional durante el terremoto de Niigata, 1964 (Ishihara y Yamazaki, 1980) y b) mediciones de ciclos de velocidad producidos por el tráfico (Huber, 1988)

Para estimar las deformaciones permanentes en los suelos, Niemunis et al. (2005), han desarrollado el modelo de acumulación de gran número de ciclos, HCA (High-Cycle Accumulacion model). Este modelo permite predecir la acumulación de deformaciones sin tener la necesidad de trazar el recorrido de la deformación para cada ciclo individual, lo que permite ahorrar tiempo y procesos de cálculo cuando se aplica en modelos de elementos finitos. La acumulación de la deformación depende de la amplitud de deformación, del actual estado del suelo, de la precarga cíclica (número de ciclos en el pasado) entre otros factores (Wichtmann et al., 2011).

A continuación se presenta un estudio de la amplitud para ciclos de deformación multidimensional convexos. Luego se plantea una posible interpretación para ciclos de deformación más complejos, lo que se estudia mediante ensayos triaxiales cíclicos drenados. Se presentan los resultados obtenidos para cargas cíclicas bidimensionales a las que son sometidas muestras de suelo no cohesivo en los que se varía simultánea y cíclicamente la carga lateral y vertical produciendo trayectorias de tensiones complejas.

La definición tensorial de amplitud para un ciclo de deformación multidimensional que está incorporado en el modelo HCA (Niemunis et al., 2005), fue propuesto originalmente por Niemunis (2003). Esta definición es aplicable solamente a ciclos de deformación convexos. La Figura 2 ejemplifica las múltiples proyecciones que un ciclo de deformación pasando del caso tridimensional al caso unidimensional.

La tasa de acumulación de deformación es aproximadamente proporcional al cuadrado de la amplitud de deformación (Wichtmann, 2005). Esta dependencia ha sido implementada en el modelo HCA (Niemunis et al., 2005) y ha sido comprobada experimentalmente para el caso bidimensional (Wichtmann et al., 2007). A diferencia de los ciclos de deformación convexos, los ciclos de deformación complejos como los mostrados en la Figura 1 y 3 , aún no tienen una interpretación adecuada en modelos como el HCA.

Proyección de $\varepsilon(\mathrm{t})$ de $3 \mathrm{D}$ a 2D

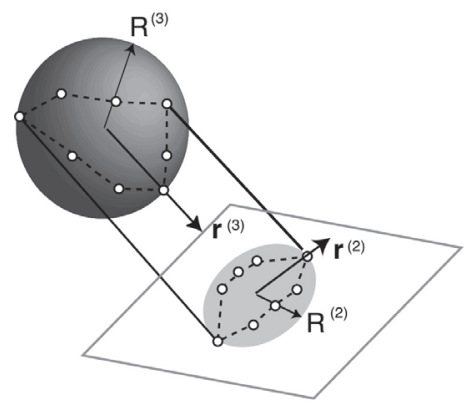

Proyección de $\varepsilon(\mathrm{t})$ de 2D a 1D

Figura 2: Proyecciones de un ciclo de deformación tridimensional al caso plano y del caso plano al caso unidimensional (Niemunis, 2005)

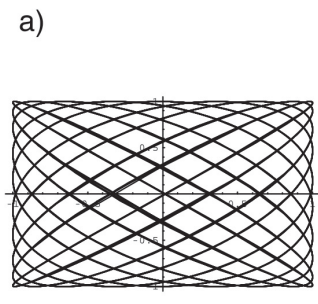

c)

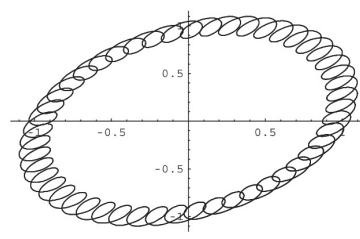

b)

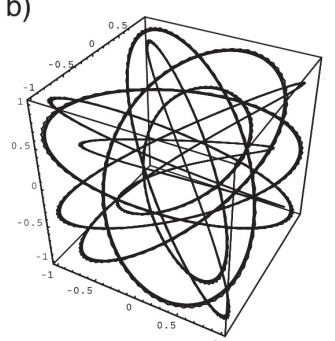

d)

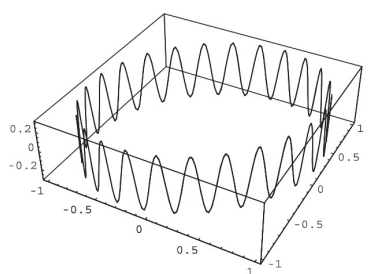

Figura 3: Ciclos de deformación complejos obtenidos mediante superposición de funciones sinusoidales: a), b) con variaciones pequeñas de la frecuencia y amplitudes y c), d), con grandes variaciones en sus frecuencias y amplitudes (Poblete y Wichtmann, 2008)

Una forma de enfrentar el problema de definir este tipo de ciclos multidimensionales complejos es el propuesto por Niemunis et al. (2007), que consiste en considerar la trayectoria de deformación como la superposición de oscilaciones armónicas individuales en las que se varían sus frecuencias. Para comprobar tal definición para el caso bidimensional se realizaron ensayos triaxiales cíclicos drenados con variación simultánea de las tensiones axial y lateral. Variando las tensiones inducidas mediante funciones armónicas con diferentes amplitudes se obtienen 
ciclos de deformación multidimensionales complejos. La Figura 4 muestra los ciclos bidimensionales complejos aplicados a los ensayos triaxiales.

(a)

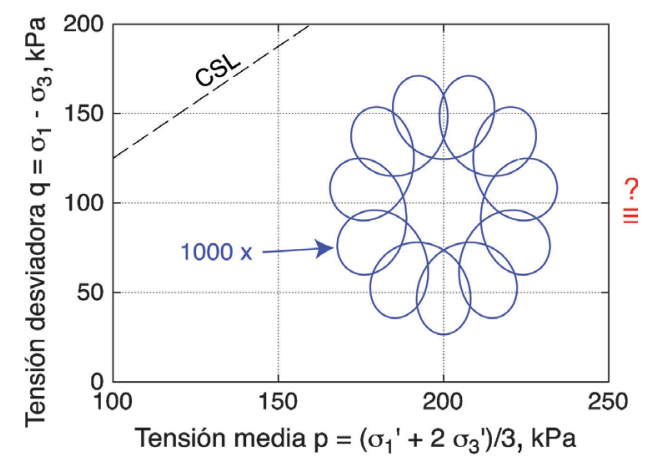

(b)

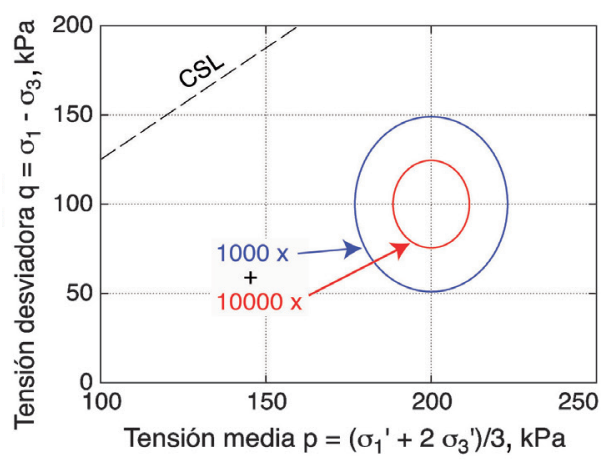

Figura 4: a) Ciclos de tensión bidimensionales y b) descomposición en oscilaciones con diferentes amplitudes

En la Figura 4a, 1000 ciclos de la trayectoria bidimensional compleja equivaldrían a la superposición de 1000 ciclos de la trayectoria elíptica (azul) de mayor amplitud y 10000 ciclos de la trayectoria elíptica de menor amplitud (rojo) en la Figura $4 b$.

La secuencia de aplicación de las oscilaciones se varió ya que se trabaja siempre con muestras de arena recién preparadas. Se demuestra experimentalmente que el orden en la aplicación de los ciclos de carga no influye en la deformación acumulada final.

\section{Resultados de los ensayos}

Se presentan una serie de ensayos realizados a muestras remoldeadas de arena fina. Se prefiere el uso de una arena fina debido a que con esto se minimiza el efecto de penetración de la membrana (Wichtmann et al., 2013). La forma de los granos es subangular y los índices de vacíos máximo y mínimo son $e_{\max }=1.054$ y $e_{\min }=0.677$ respectivamente. La gravedad específica de esta arena alcanza un valor de $G_{S}=2.65$. Se preparan las muestras mediante el método de lluvia de arena con densidades relativas iniciales de aproximadamente $\mathrm{DR}=50 \%$. Las muestras cilíndricas de diámetro $d=10 \mathrm{~cm}$ y altura $h=20$ $\mathrm{cm}$ se saturan con $\mathrm{CO}_{2}$ y con agua desaireada. Para todos los ensayos se utiliza una contrapresión de $500 \mathrm{kPa}$. La frecuencia y el número de ciclos de cada ensayo varía entre los 0.2 y $0.5 \mathrm{~Hz}$ y entre los 1000 y 10000 ciclos. La Figura 5 a muestra la curva granulométrica de la arena utilizada. (a)

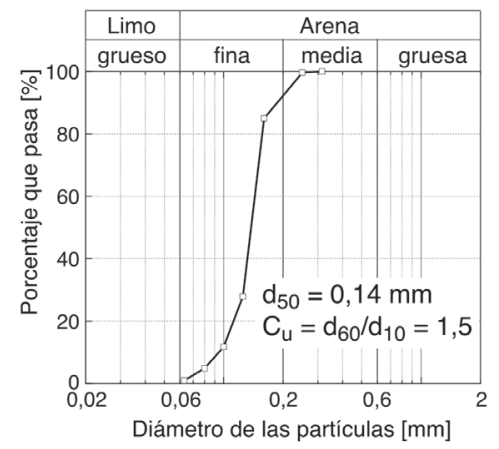

(b)

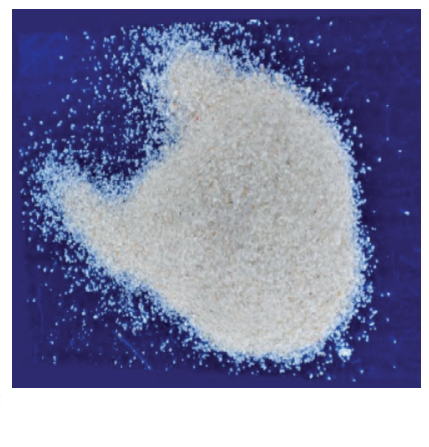

Figura 5: a) Distribución granulométrica y b) fotografía de la arena utilizada

La Figura 6 muestra un esquema del equipo triaxial cíclico de carga controlada, mediante el cual se pueden aplicar ciclos de carga definidos por el usuario. Se aplican ciclos de carga sinusoidales tanto verticales como horizontales. Esta combinación de cargas simultáneas permite obtener trayectorias complejas de tensión como las que se observan en las Figuras 4, 7 y 8.

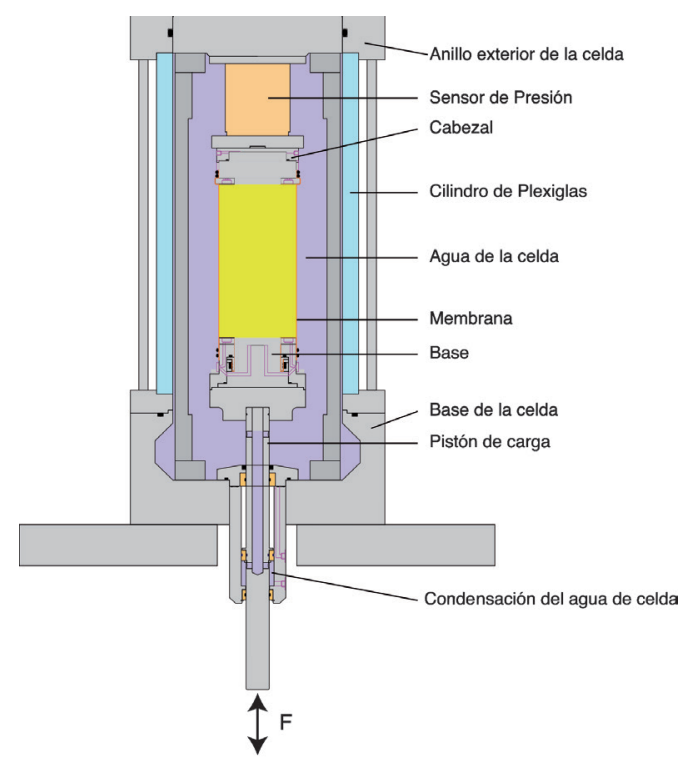

Figura 6: Esquema del equipo triaxial cíclico de carga vertical y horizontal controlada por el usuario. 
La Figura 7 presenta las trayectorias de tensiones de los ensayos realizados en el plano isomorfo $P$ - $Q$, donde $P=$ $\sqrt{3} p$ y $Q=\sqrt{2 / 3} q$ son variables isomorfas (Niemunis, 2003). El uso de estas variables es ventajoso ya que al pasar de un sistema coordenado de tensiones principales al plano $P-Q$, la longitud y los ángulos de las trayectorias de tensiones se mantienen. La Figura 7 a muestra una trayectoria compleja en forma de flor, la cual se repite 1000 veces. Un ciclo de carga complejo consiste en recorrer la trayectoria indicada en forma completa. La Figura $7 \mathrm{~b}$ muestra la descomposición como la suma de 1000 ciclos de mayor amplitud (en rojo), y 10000 ciclos de menor amplitud (en azul). El ensayo representado por la Figura $7 \mathrm{~b}$ se repite invirtiendo el orden de aplicación de la carga. A modo de comparación se dibuja también en la Figura 7b la trayectoria compleja en color gris.

(a)

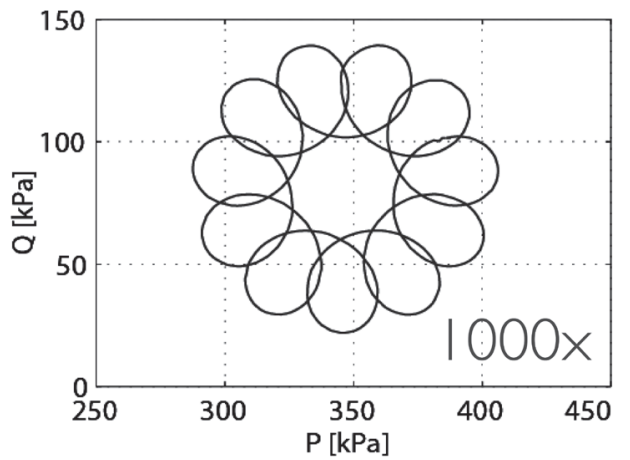

(b)

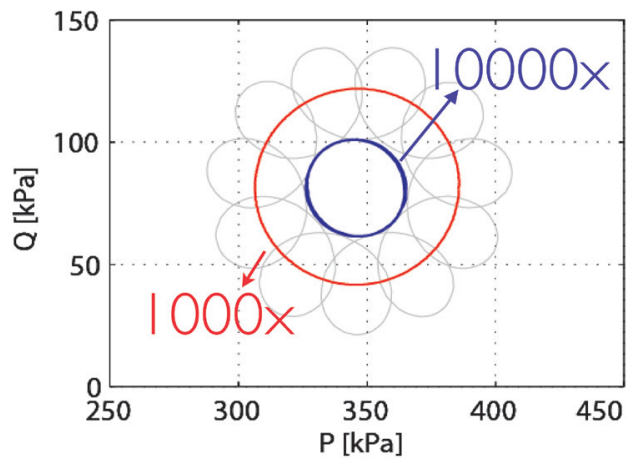

Figura 7: Trayectorias de tensiones de los ensayos realizados en el espacio isomorfo $P-Q$ (donde $P=\sqrt{3} p$ y $Q=\sqrt{2 / 3} q$, a) trayectoria compleja en forma de flor y b) descomposición de las trayectorias con diferentes frecuencias

La variación cíclica de la tensión vertical y radial para la trayectoria compleja se muestra en la Figura 8 donde las ecuaciones (1) y (2) representan dicha variación. Estas tensiones se aplican simultáneamente variando cíclicamente las tensiones totales vertical $\sigma_{\mathrm{Z}}$ y radial $\sigma_{\mathrm{R}}$. Las expresiones (1) y (2) muestran el tipo de ecuación utilizada para definir estas trayectorias complejas de tensión, donde $t$ es el tiempo.

$$
\begin{aligned}
& P(t)=200 \sqrt{3}+40 \cos \left(\frac{\pi t}{100}\right)+20 \operatorname{sen}\left(\frac{\pi t}{10}\right) \\
& Q(t)=100 \sqrt{2 / 3}+20 \cos \left(\frac{\pi t}{10}\right)+40 \operatorname{sen}\left(\frac{\pi t}{100}\right)
\end{aligned}
$$

(a)

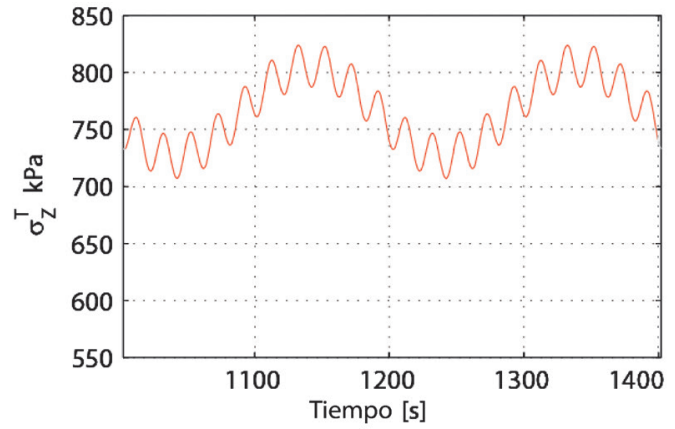

(b)

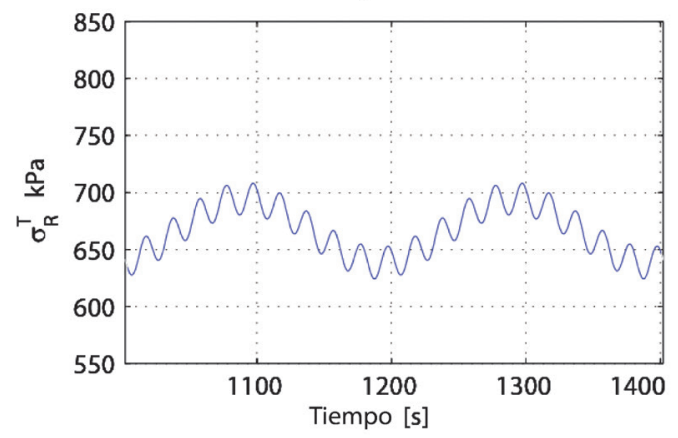

Figura 8: Tensiones totales a) axial y b) radial aplicadas simultáneamente en el ensayo triaxial cíclico de carga controlada para trayectoria compleja

Para cada ciclo de carga se registra la deformación cíclica obtenida midiendo la deformación acumulada al final de cada ciclo como se muestra en la Figura 9. Las deformaciones acumuladas vertical $\varepsilon_{1}$, volumétrica $\varepsilon_{\mathrm{V}}$, desviadora $\varepsilon_{\mathrm{q}}$, total $\varepsilon_{\mathrm{T}}$, y horizontal $\varepsilon_{3}$, se presentan en la Figura 10 en función del número de ciclos $\mathrm{N}$ tanto en escala lineal como logarítmica.

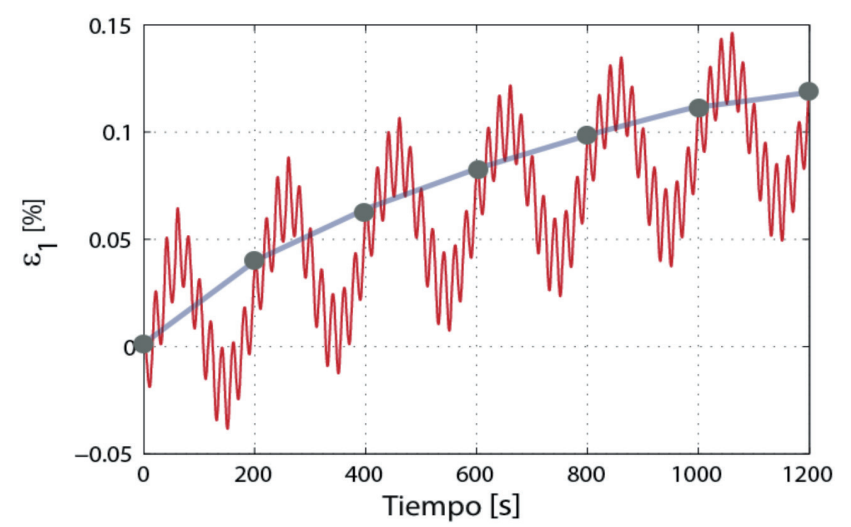

Figura 9: Registro de la deformación acumulada al final de cada ciclo. La línea roja muestra la deformación actual y los puntos la deformación acumulada. 
(a)

(b)
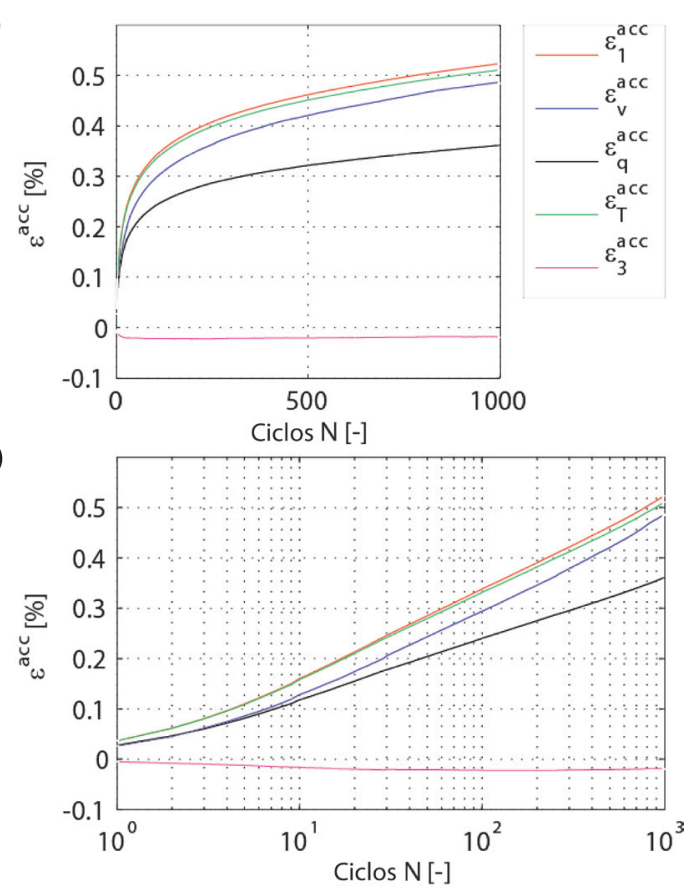

Figura 10: a) Deformación acumulada como función del número de ciclos para una densidad relativa $\mathrm{DR}=50 \%$ y b) en escala logarítmica

(a)

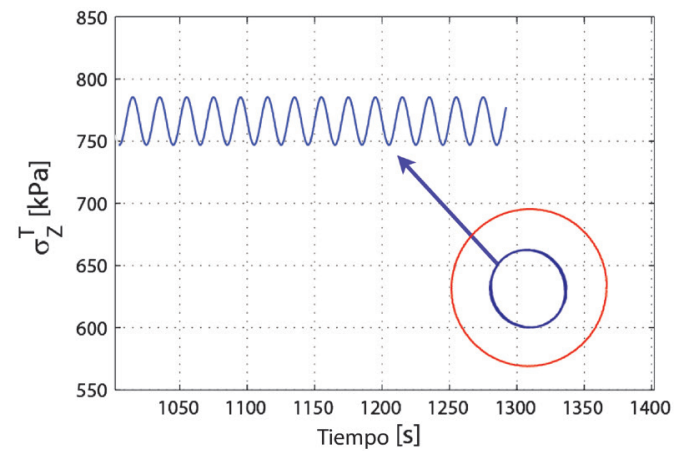

(b)

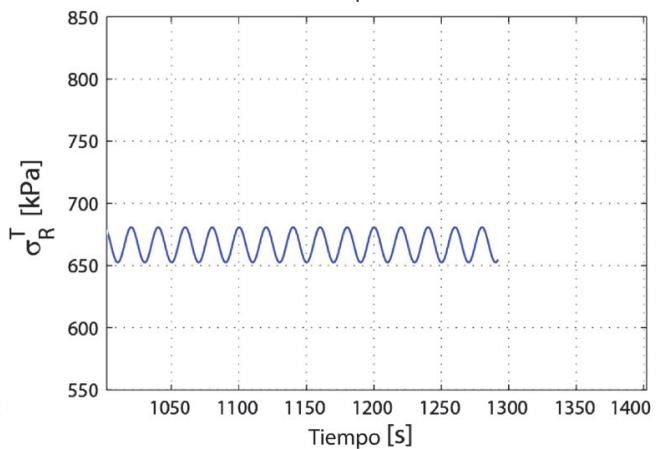

Figura 11: Tensiones totales a) axial y b) radial aplicadas simultáneamente en el ensayo triaxial cíclico de carga controlada para trayectoria simple circular de menor amplitud

La descomposición de la trayectoria de tensiones compleja se realiza en dos fases con trayectorias simples circulares (en el espacio isomorfo). En primer lugar se somete la muestra a 10000 ciclos de menor amplitud (Figura 11), para luego y en la misma probeta aplicar 1000 ciclos de mayor amplitud (Figura 12). Las funciones de cada una de las fases corresponden a la separación de las ecuaciones (1) y (2).

(a)

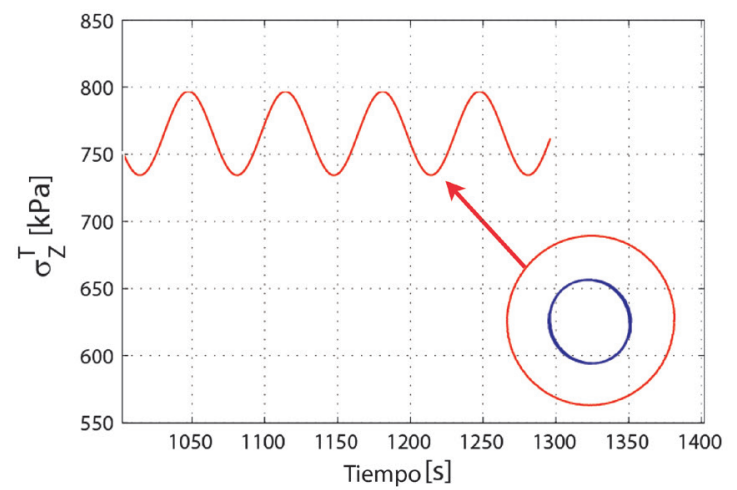

(b)

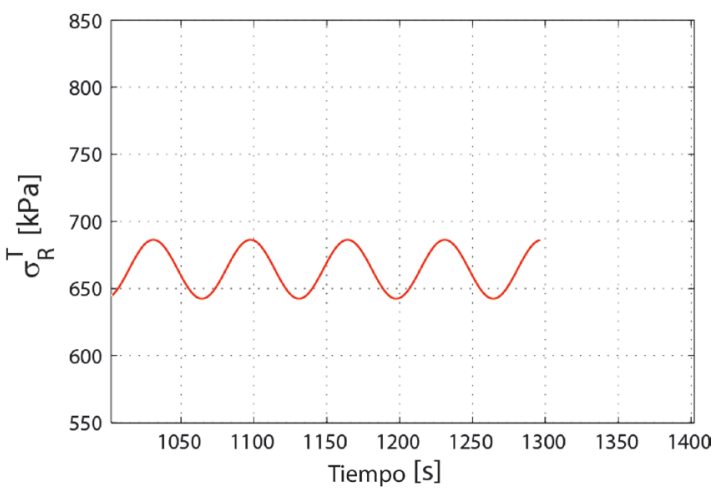

Figura 12: Tensiones totales a) axial y b) radial aplicadas simultáneamente en el ensayo triaxial cíclico de carga controlada para trayectoria simple circular de mayor amplitud.

(a)

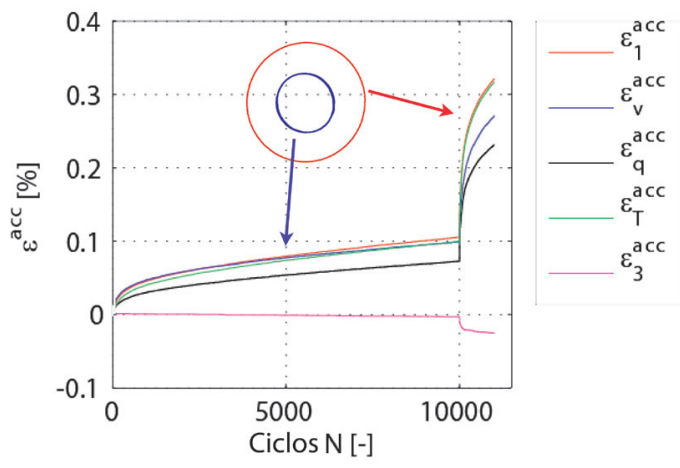

(b)

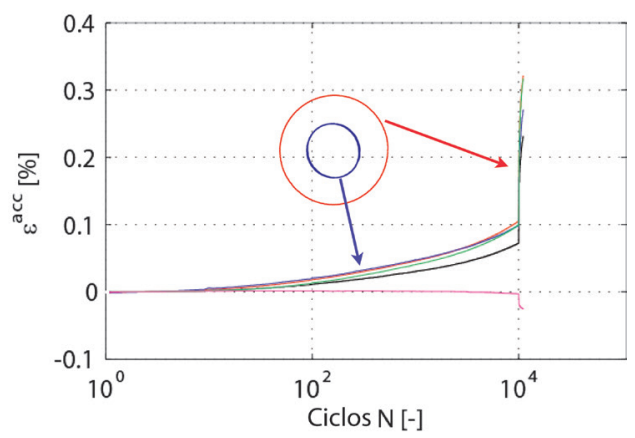

Figura 13: a) Deformación acumulada como función del número de ciclos y b) escala logarítmica 
Al igual que para la trayectoria compleja, se registra la deformación cíclica obtenida midiendo la deformación acumulada al final de cada ciclo. Las deformaciones acumuladas se presentan en la Figura 13 tanto en escala lineal como logarítmica. El salto en la deformación acumulada que se produce a los 10000 ciclos se debe al cambio de la amplitud de tensión inducida.

La Figura 14 muestra el resumen de los casos ensayados. Se puede observar que de la trayectoria compleja en forma de flor se obtiene una acumulación de deformación mayor que la de descomposición en trayectorias simples. También se puede observar que la acumulación de deformación es independiente del orden de aplicación de las oscilaciones, pudiéndose aplicar la regla de Miner (1945), para ciclos de deformación circulares. La diferencia en la acumulación puede deberse a la diferencia que existe en la ubicación de la trayectoria de menor amplitud ya que para el caso descompuesto los 10000 ciclos se aplican en el mismo lugar a diferencia del caso complejo en el cual la trayectoria recorre diferentes zonas del diagrama $P-Q$.

(a)

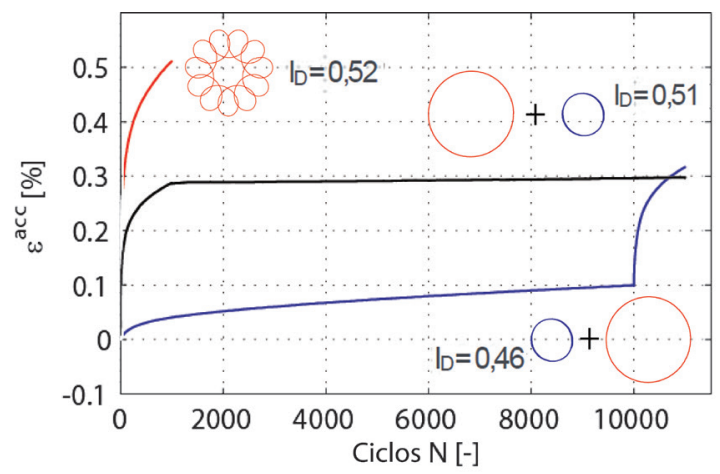

(b)

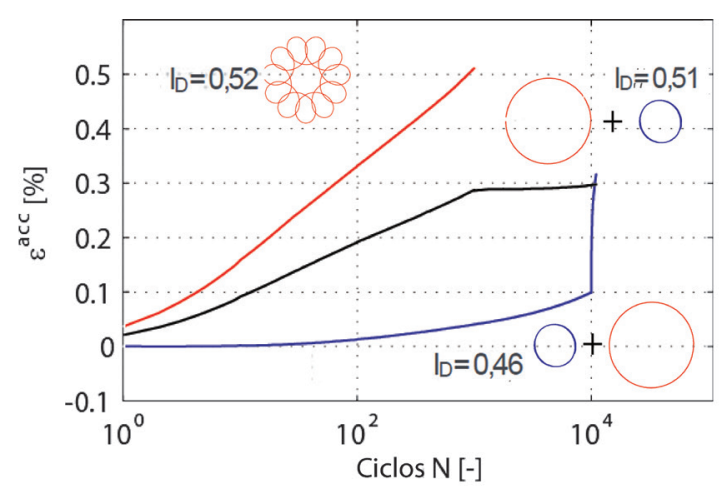

Figura 14: Comparación de la deformación acumulada para la trayectoria compleja y su descomposición

\section{Conclusiones}

Se presenta evidencia experimental que ratifica la importancia de la forma de los ciclos de deformación en el porcentaje de acumulación de deformaciones para el caso de carga cíclica drenada. Se utiliza experimentalmente un nuevo método para determinar la acumulación de deformación que consiste en la descomposición de las trayectorias complejas en una serie de oscilaciones armónicas de diferente frecuencia. Sin embargo, se determina que la acumulación de deformación obtenida con la descomposición es menor que la obtenida al separar las oscilaciones. Para poder ratificar o descartar el método de descomposición se requiere la realización de una mayor cantidad de ensayos variando la forma, posición y dimensión de los ciclos aplicados.

\section{Agradecimientos}

El primer autor agradece los aportes del DAAD y CONICYT en el marco del programa de postgrado Chile-Alemania 2006-2010 (beca de doctorado número: 07-DOCDAAD-12). También el primer autor agradece el apoyo brindado en la realización de los ensayos de laboratorio en el Instituto de Mecánica de Suelos y Mecánica de Rocas IBF del Instituto de Tecnología de Karlsruhe KIT en Alemania.

\section{Referencias}

Huber, G. (1988). Erschütterungsausbreitung beim Rad/ Schiene-System. PhD thesis, Institute of Soil Mechanics and Rock Mechanics, University of Karlsruhe, Germany, Issue No. 115 (en alemán)

Ishihara, K. and Yamazaki, F. (1980). Cyclic simple shear tests on saturated sand in multi-directional loading. Soils and Foundations 20, No. 1, 45-59

Miner, M. (1945). Cumulative damage in fatigue. Journal of Applied Mechanics 12(3), 159-164

Niemunis. A. (2003). Extended hypoplastic models for soils. Habilitation, Ruhr-University Bochum, Germany, Issue No. 34, disponible en www.pg.gda.pl/ aniem/an-liter.html.

Niemunis, A., Wichtmann, T. and Triantafyllidis, Th. (2005). A high-cycle accumulation model for sand. Computers and Geotechnics 32, No. 4, 245-263 
Niemunis, A., Wichtmann, T. and Triantafyllidis, Th. (2007). Wichtmann. T. (2005). Explicit accumulation model for nonOn the definition of the fatigue loading for sand. International cohesive soils under cyclic loading. Ruhr-University Bochum, Workshop on Constitutive Modelling - Development, Germany, Issue No. 38 Implementation, Evaluation, and Application, 12-13 January 2007, Hong Kong.

Wichtmann, T., Niemunis, A. and Triantafyllidis, Th. (2011). Prediction of drained and undrained cyclic behaviour of a fine

Poblete, M. and Wichtmann, T. (2008). Accumulation of residual sand using a high-cycle accumulation model. $5^{\text {th }}$ International deformations due to cyclic loading with complicated strain loops. Obras y Proyectos 4, 44-52

Conference on Earthquake Geotechnical Engineering, Santiago, Chile

Wichtmann, T., Niemunis, A. and Triantafyllidis, Th. (2007). On the influence of the polarization and the shape of the strain loop on strain accumulation in sand under high-cyclic loading. Soil Dynamics and Earthquake Engineering 27, No. 1, 14-28

Wichtmann, T., Niemunis, A. and Triantafyllidis, Th. (2013). On the "elastic stiffness" in a high-cycle accumulation model - continued investigations. Canadian Geotechnical Journal 50(12), 1260-1272 